\title{
The Formation of Fullerenes in Planetary Nebulae
}

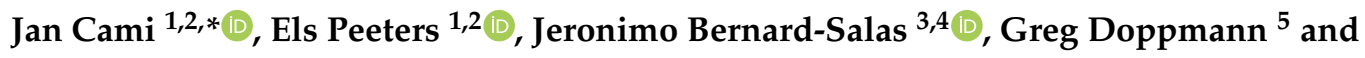 \\ James De Buizer 6 \\ 1 Department of Physics and Astronomy and Centre for Planetary Science and Exploration (CPSX), \\ The University of Western Ontario, London, ON N6A 3K7, Canada; epeeters@uwo.ca \\ 2 SETI Institute, 189 Bernardo Ave, Suite 100, Mountain View, CA 94043, USA \\ 3 Robert Hooke Building, Department of Physical Sciences, The Open University, \\ Milton Keynes MK7 6AA, UK; Jeronimo.Bernard-Salas@open.ac.uk \\ 4 ACRI-ST, 260 Route du Pin Montard, 06904 Sophia-Antipolis, France \\ 5 W. M. Keck Observatory, 65-1120 Mamalahoa Highway, Kamuela, HI 96743, USA; \\ gdoppmann@keck.hawaii.edu \\ 6 Stratospheric Observatory for Infrared Astronomy-USRA, NASA Ames Research Center, MS N232-12, \\ Moffett Field, CA 94035, USA; jdebuizer@sofia.usra.edu \\ * Correspondence: jcami@uwo.ca; Tel.: +1-519-661-2111 (ext. 80978)
}

Received: 31 July 2018; Accepted: 18 September 2018; Published: 21 September 2018

\begin{abstract}
In the last decade, fullerenes have been detected in a variety of astrophysical environments, with the majority being found in planetary nebulae. Laboratory experiments have provided us with insights into the conditions and pathways that can lead to fullerene formation, but it is not clear precisely what led to the formation of astrophysical fullerenes in planetary nebulae. We review some of the available evidence, and propose a mechanism where fullerene formation in planetary nebulae is the result of a two-step process where carbonaceous dust is first formed under unusual conditions; then, the fullerenes form when this dust is being destroyed.
\end{abstract}

Keywords: planetary nebulae; fullerenes

\section{Introduction}

When Kroto et al. [1] conducted a series of experiments to simulate the chemistry occurring in the surroundings of carbon-rich evolved stars, they discovered a new and particularly stable carbonaceous molecule: Buckminsterfullerene, $C_{60}$. We now know that $C_{60}$ is the most stable (and best known) member of an entire class of large, cage-like carbonaceous molecules. Given the stability of the molecule and the nature of the simulation experiments, Kroto et al. [1] immediately concluded that $C_{60}$ was most likely widespread and abundant in space, and as soon as spectroscopic data were available, astronomers searched for its telltale signature in interstellar and circumstellar environments see [2].

We can now confirm that $\mathrm{C}_{60}$ is indeed widespread and abundant in space. Since the first unambiguous detection of all IR active vibrational modes of $\mathrm{C}_{60}$ in the Spitzer-IRS spectrum of the planetary nebula (PN) Tc 1 at 7.0, 8.5, 17.4, and $18.9 \mu \mathrm{m}$ [3], the same spectral features have been found in a variety of evolved star environments (see Section 3), as well as in Reflection Nebulae RNe; see e.g., [4,5], the diffuse ISM [6], and young stellar objects and Herbig Ae/Be stars [7]. Recently, laboratory experiments and astronomical observations have confirmed the identification of two strong (and 3 weaker) diffuse interstellar bands DIBs; see [8] as due to electronic transitions of the $C_{60}^{+}$cation see e.g., [9-12], and references therein. It is estimated that, on average, $C_{60}$ locks up $10^{-4}-10^{-3}$ of the cosmic carbon $[9,13,14]$, a considerable abundance for a single species!

While it is thus established that $\mathrm{C}_{60}$ is abundantly present in interstellar and circumstellar environments, the life cycle of the $\mathrm{C}_{60}$ molecule itself is not crystal clear. Here, we review what we 
know about the conditions that lead to the formation of fullerenes and how this can be reconciled with the body of available observational evidence to construct a coherent formation mechanism for fullerenes in evolved star environments.

\section{The Conditions Required to form Fullerenes}

Laboratory experiments (as well as theoretical calculations) on the condensation of carbonaceous gas have provided important information about the carbon chemistry that can occur in circumstellar environments. The key parameter that determines the outcome is the temperature (see Figure 1 for a schematic overview). At low temperatures, the condensation products are a variety of (small) molecules (e.g., $\mathrm{C}_{2} \mathrm{H}_{2}, \mathrm{HCN}$...); any dust that condenses out will be primarily in the form of amorphous carbon [15]. Above $\sim 1000 \mathrm{~K}$, the nature of the possible chemical reactions changes, and now benzene can form through a series of chemical reactions and from this species, a whole family of polycyclic aromatic hydrocarbon (PAH) molecules [16,17]. These PAHs are then the condensation nuclei for the formation of soot; the soot is formed quickly, and is graphitic in nature [17]. Finally, similar experiments at much higher temperatures (above $\sim 3500 \mathrm{~K}$ ) result in the formation of fullerene molecules in addition to a soot that is fullerenic in nature [17]. Thus, different temperatures result in distinctly different products: no fullerenes are formed at the PAH-favoured temperatures, and similarly no PAHs appear in the high temperature experiments. This is partly due to hydrogen atoms that inhibit the formation of fullerenes at the lower temperatures; when similar experiments are carried out in H-poor conditions, fullerenes do form at these lower temperatures [18].

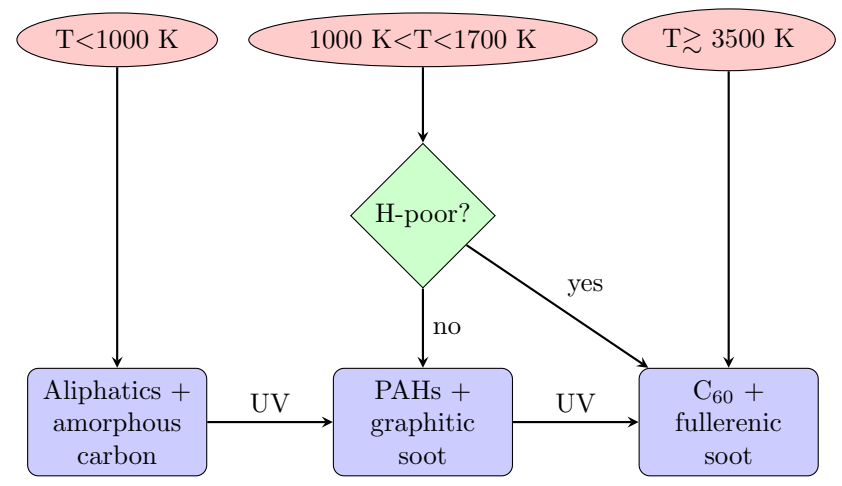

Figure 1. Formation routes for carbonaceous materials based on [15-18].

Irradiation of these carbonaceous compounds by UV photons can then significantly change their nature. It has long been known that UV irradiation of amorphous carbon leads to aromatization in the compounds. More recently, theoretical calculations [13,19] and laboratory experiments [20] have shown that large PAHs are first stripped of their $\mathrm{H}$ atoms by UV photons; the resulting pure graphene flake then curls up and shrinks by emission of $C_{2}$ units until it reaches the most stable configuration $-\mathrm{C}_{60}$. It is thus conceivable that a significant amount of carbonaceous dust can be transformed by these processes over their lifetime-from aliphatic to aromatic to fullerenic material.

\section{Observations of Fullerenes in PNe}

While the IR $\mathrm{C}_{60}$ bands have been detected in various environments, the majority of detections originates from evolved stars (including post-AGB and pre-PN objects, but primarily in PNe in the Milky Way and the Magellanic Clouds; see e.g., [21-23] and references therein). All $\mathrm{C}_{60}-\mathrm{PNe}$ have very similar properties and IR spectra. Indeed, they are all fairly young, low-excitation objects, the IR spectra of which are characterized by a rising, featureless continuum on which several emission features are superposed: The $\mathrm{C}_{60}$ bands, a strong 11-13 $\mu \mathrm{m}$ emission plateau (possibly due to 
SiC), a 6-9 $\mu \mathrm{m}$ emission plateau, and a strong $30 \mu \mathrm{m}$ feature [22,23]. Thus, the conditions that are conducive to the formation and excitation of $\mathrm{C}_{60}$ also result in the appearance of other dust components. Notably, PAH emission in these objects is very weak or absent [23]. When comparing optical and near-IR colors of these objects to other evolved stars, the fullerene sources are the least reddened objects (i.e., the direct lines of sight to the central stars do not contain much dust that can cause reddening) and cluster together in near-IR color-color diagrams, while other evolved objects have a large range in colors [24].

Spatial studies of the different emission components can give clues to the conditions and origin of the different components. Narrow-band images of Tc 1 clearly show different distributions of the ionized gas, the dust responsible for the continuum emission, and the fullerenes (see Figure 2). They reveal that the fullerenes emit in a ring $\sim 5^{\prime \prime}$ away from the central star, at the interface between the ionized region and the surrounding Photo-Dissociation Region (PDR). There is no clear evidence for PAHs in this object, but if a very weak $11.3 \mu \mathrm{m}$ bump is to be associated with PAHs, then those are all located in the top part of the central horse-shoe shaped region associated with the continuum dust emission. In that case, the PAHs and fullerenes would clearly be spatially separated, with the fullerenes at a location where the radiation field should be weaker than at the location of the PAHs.

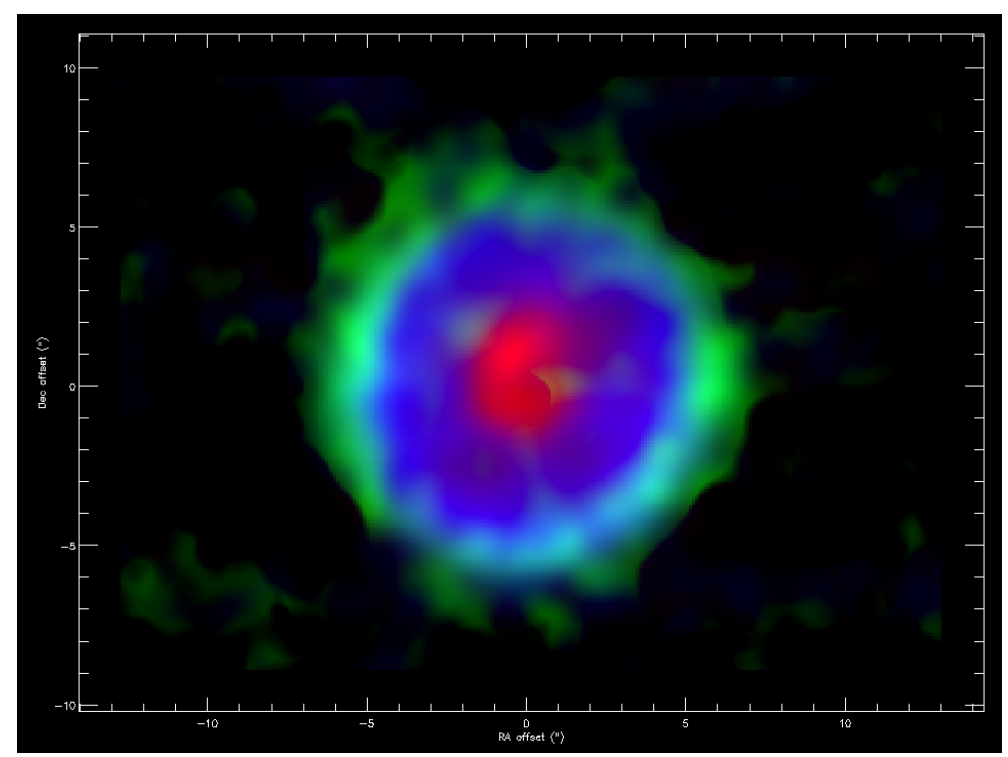

Figure 2. Decomposition of Gemini/T-ReCS narrow-band infrared images of Tc 1 into the different components (Cami et al., in prep): the continuum dust emission (red), the ionized gas (blue), and the fullerene emission (green).

Finally, an important observational result is that the $\mathrm{C}_{60}$ detection rate in PNe is fairly low: Only 3-5\% of the Galactic PNe observed by Spitzer-IRS at high resolution exhibit the $\mathrm{C}_{60}$ bands [23]; the fraction of $\mathrm{C}_{60}$ detections in the Magellanic Clouds is slightly higher [22]. These low rates imply that the $\mathrm{C}_{60}$ molecules either form in a small fraction of objects (possibly related to requiring specific formation and/or excitation conditions), or alternatively that pure $\mathrm{C}_{60}$ has only a short lifetime in these environments, possibly related to chemically altering the molecular structure that would significantly affect the spectroscopic signatures.

\section{Discussion}

The circumstellar environments of evolved stars represent what happened to an atomic gas (the stellar envelope) that cooled after ejection due to mass loss processes. When considering the formation of molecules and dust in these environments, we thus always start from bottom up processes. In most cases (for typical condensation temperatures), we would expect the end products in C-rich 
environments to be PAHs and graphitic dust. Only when the temperatures are high or the environment is $\mathrm{H}$-poor would fullerenes and fullerenic dust result.

The most straightforward scenario for the formation of fullerenes is thus one where the $\mathrm{C}_{60}-\mathrm{PNe}$ represent objects where-for some reason-the condensation happens at higher temperatures or under $\mathrm{H}$-poor conditions. This must have happened close to the central star (otherwise temperatures and densities would be too low), and consequently the condensation products moved outward since its formation-at least for Tc 1 where we see the $\mathrm{C}_{60}$ emission far from the central star. Since the formation of $\mathrm{C}_{60}$ also results in large amounts of fullerenic soot, while no dust is seen at the location of the $\mathrm{C}_{60}$ emission, dust destruction must have occurred as well. This would then explain why the colors of the $\mathrm{C}_{60}-\mathrm{PNe}$ are so similar and appear unreddened. Perhaps the $\mathrm{C}_{60}$ molecules are the only species to survive this process, but it is conceivable that the destruction of fullerenic dust actually results in the formation of $\mathrm{C}_{60}$ molecules, e.g., by "liberating" $\mathrm{C}_{60}$ molecules from the dust grains. This dust destruction process must then be related to the transition of a post-AGB object to a PN since we only see the $\mathrm{C}_{60}$ bands in young PNe. Shock waves related to a fast wind overtaking a slow wind, or the development of an ionization front appear to be the most likely direct causes. The formation of $\mathrm{C}_{60}$ in PNe would thus involve a two-step process: Condensation (in an earlier phase, likely the AGB) under unusual conditions to produce $\mathrm{C}_{60}$ and fullerenic dust, followed by the destruction of the dust (that possibly creates more $\mathrm{C}_{60}$ ). Such a scenario can explain why the $\mathrm{C}_{60}$-PNe have the observational properties they have.

There is of course a fundamentally different formation route for $\mathrm{C}_{60}$ that we should also consider. Given that the central stars of PNe are hot stars that produce copious amounts of UV photons, photo-processing of carbonaceous materials seems like a plausible way to produce fullerenes, and indeed, it has been suggested that the formation of $\mathrm{C}_{60}$ is the result of photo-processing of hydrogenated amorphous carbon (HAC) dust grains or similar carbon clusters or PAHs see e.g., [21,25]. However, if this process can efficiently convert carbonaceous dust to fullerenes in the low-excitation $\mathrm{C}_{60}-\mathrm{PNe}$, the same process should be even more efficient in the more mature objects where timescales for processing have been longer, and UV photons are more abundant. Similarly, much of the dust in Tc 1 would have been converted to fullerenes as well, since it is located five times closer to the source of UV photons than the fullerenes themselves. In other words, if photo-processing of HACs (or even PAHs) would be the general process for fullerene formation in young PNe, there should only be very few PNe left that have circumstellar PAHs or in fact any carbonaceous material at all other than fullerenes.

The only remaining question is then why the dust condensation conditions were different in the $\mathrm{C}_{60}-\mathrm{PNe}$, and this may be tied to the evolutionary history of the central stars. For about half of the $\mathrm{C}_{60}-\mathrm{PNe}$, it has been established that their central stars are of [WC] type see e.g., [23]; certainly for such objects, it is plausible that condensation could happen in H-poor environments that would be more suited to create fullerenes and fullerenic soot than other objects. It is not clear what the status of the other $\mathrm{C}_{60}-\mathrm{PNe}$ is in that sense, and we should also point out that not all PNe with [WC] stars display the fullerenes. Future observations that allow to measure e.g., the carbon abundance would be crucial to determine the underlying cause for the different condensation conditions.

Author Contributions: Conceptualization, J.C., E.P. and J.B.-S.; Data curation, J.D.B.; Formal analysis, J.C.; Funding acquisition, J.C., E.P. and G.D.; Methodology, J.C.; Software, J.C.; Visualization, J.C.; Writing-original draft, J.C.; Writing-review \& editing, J.C., E.P., J.B.-S. and G.D.

Funding: Natural Sciences and Engineering Research Council of Canada: RGPIN-2016-06047.

Acknowledgments: J.C. and E.P. acknowledge support from an NSERC DG.

Conflicts of Interest: The authors declare no conflict of interest. 


\section{References}

1. Kroto, H.W.; Heath, J.R.; Obrien, S.C.; Curl, R.F.; Smalley, R.E. C60: Buckminsterfullerene. Nature 1985, 318, 162-163. [CrossRef]

2. Herbig, G.H. The Search for Interstellar C 60 . Astrophys. J. 2000, 542, 334-343. [CrossRef]

3. Cami, J.; Bernard-Salas, J.; Peeters, E.; Malek, S.E. Detection of $\mathrm{C}_{60}$ and $\mathrm{C}_{70}$ in a Young Planetary Nebula. Science 2010, 329, 1180-1182. [CrossRef] [PubMed]

4. Sellgren, K.; Werner, M.W.; Ingalls, J.G.; Smith, J.D.T.; Carleton, T.M.; Joblin, C. C60 in Reflection Nebulae. Astrophys. J. Lett. 2010, 722, L54-L57. [CrossRef]

5. Peeters, E.; Tielens, A.G.G.M.; Allamandola, L.J.; Wolfire, M.G. The 15-20 $\mu \mathrm{m}$ Emission in the Reflection Nebula NGC 2023. Astrophys. J. 2012, 747, 44. [CrossRef]

6. Berné, O.; Cox, N.L.J.; Mulas, G.; Joblin, C. Detection of buckminsterfullerene emission in the diffuse interstellar medium. Astron. Astrophys. 2017, 605, L1. [CrossRef] [PubMed]

7. Roberts, K.R.G.; Smith, K.T.; Sarre, P.J. Detection of $\mathrm{C}_{60}$ in embedded young stellar objects, a Herbig Ae/Be star and an unusual post-asymptotic giant branch star. Mon. Not. R. Astron. Soc. 2012, 421, 3277-3285. [CrossRef]

8. Foing, B.H.; Ehrenfreund, P. Detection of Two Interstellar Absorption Bands Coincident with Spectral Features of $\mathrm{C}_{60}^{+}$. Nature 1994, 369, 296. [CrossRef]

9. Campbell, E.K.; Holz, M.; Gerlich, D.; Maier, J.P. Laboratory confirmation of $\mathrm{C}_{60}^{+}$as the carrier of two diffuse interstellar bands. Nature 2015, 523, 322-323. [CrossRef] [PubMed]

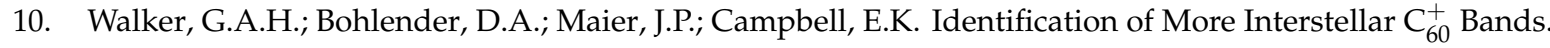
Astrophys. J. Lett. 2015, 812, L8. [CrossRef]

11. Walker, G.A.H.; Campbell, E.K.; Maier, J.P.; Bohlender, D. The 9577 and 9632 A Diffuse Interstellar Bands: $\mathrm{C}_{60}^{+}$as Carrier. Astrophys. J. 2017, 843, 56. [CrossRef]

12. Cordiner, M.A.; Cox, N.L.J.; Lallement, R.; Najarro, F.; Cami, J.; Gull, T.R.; Foing, B.H.; Linnartz, H.; Lindler, D.J.; Proffitt, C.R.; et al. Searching for Interstellar $\mathrm{C}_{60}^{+}$Using a New Method for High Signal-to-noise HST/STIS Spectroscopy. Astrophys. J. Lett. 2017, 843, L2. [CrossRef]

13. Berné, O.; Tielens, A.G.G.M. Formation of buckminsterfullerene (C60) in interstellar space. Proc. Natl. Acad. Sci. USA 2012, 109, 401-406. [CrossRef] [PubMed]

14. Omont, A. Interstellar fullerene compounds and diffuse interstellar bands. Astron. Astrophys. 2016, 590, A52. [CrossRef]

15. Gail, H.P.; Sedlmayr, E. Formation of crystalline and amorphous carbon grains. Astron. Astrophys. 1984, 132, 163-167.

16. Frenklach, M.; Feigelson, E.D. Formation of polycyclic aromatic hydrocarbons in circumstellar envelopes. Astrophys. J. 1989, 341, 372-384. [CrossRef]

17. Jäger, C.; Huisken, F.; Mutschke, H.; Jansa, I.L.; Henning, T. Formation of Polycyclic Aromatic Hydrocarbons and Carbonaceous Solids in Gas-Phase Condensation Experiments. Astrophys. J. 2009, 696, 706-712. [CrossRef]

18. Wang, X.K.; Lin, X.W.; Mesleh, M.; Jarrold, M.F.; Dravid, V.P.; Ketterson, J.B.; Chang, R.P.H. The effect of hydrogen on the formation of carbon nanotubes and fullerenes. J. Mater. Res. 1995, 10, 1977-1983. [CrossRef]

19. Berné, O.; Montillaud, J.; Joblin, C. Top-down formation of fullerenes in the interstellar medium. Astron. Astrophys. 2015, 577, A133. [CrossRef] [PubMed]

20. Zhen, J.; Castellanos, P.; Paardekooper, D.M.; Linnartz, H.; Tielens, A.G.G.M. Laboratory Formation of Fullerenes from PAHs: Top-down Interstellar Chemistry. Astrophys. J. Lett. 2014, 797, L30. [CrossRef]

21. García-Hernández, D.A.; Manchado, A.; García-Lario, P.; Stanghellini, L.; Villaver, E.; Shaw, R.A.; Szczerba, R.; Perea-Calderón, J.V. Formation of Fullerenes in H-containing Planetary Nebulae. Astrophys. J. Lett. 2010, 724, L39-L43. [CrossRef]

22. García-Hernández, D.A.; Iglesias-Groth, S.; Acosta-Pulido, J.A.; Manchado, A.; García-Lario, P.; Stanghellini, L.; Villaver, E.; Shaw, R.A.; Cataldo, F. The Formation of Fullerenes: Clues from New $\mathrm{C}_{60}$, $\mathrm{C}_{70}$, and (Possible) Planar $\mathrm{C}_{24}$ Detections in Magellanic Cloud Planetary Nebulae. Astrophys. J. Lett. 2011, 737, L30. [CrossRef]

23. Otsuka, M.; Kemper, F.; Cami, J.; Peeters, E.; Bernard-Salas, J. Physical properties of fullerene-containing Galactic planetary nebulae. Mon. Not. R. Astron. Soc. 2014, 437, 2577-2593. [CrossRef] 
24. Sloan, G.C.; Lagadec, E.; Zijlstra, A.A.; Kraemer, K.E.; Weis, A.P.; Matsuura, M.; Volk, K.; Peeters, E.; Duley, W.W.; Cami, J.; et al. Carbon-rich Dust Past the Asymptotic Giant Branch: Aliphatics, Aromatics, and Fullerenes in the Magellanic Clouds. Astrophys. J. 2014, 791, 28. [CrossRef]

25. Micelotta, E.R.; Jones, A.P.; Cami, J.; Peeters, E.; Bernard-Salas, J.; Fanchini, G. The Formation of Cosmic Fullerenes from Arophatic Clusters. Astrophys. J. 2012, 761, 35. [CrossRef]

(C) 2018 by the authors. Licensee MDPI, Basel, Switzerland. This article is an open access article distributed under the terms and conditions of the Creative Commons Attribution (CC BY) license (http:/ / creativecommons.org/licenses/by/4.0/). 